\title{
Tailoring Enhanced Optical Chirality: Design Principles for Chiral Plasmonic Nanostructures
}

\author{
Martin Schäferling, Daniel Dregely, Mario Hentschel, and Harald Giessen* \\ 4th Physics Institute, Research Center SCoPE, and Research Center SimTech, University of Stuttgart, \\ Pfaffenwaldring 57, 70550 Stuttgart, Germany
}

(Received 24 August 2011; revised manuscript received 5 April 2012; published 14 August 2012)

\begin{abstract}
Electromagnetic fields with strong optical chirality can be formed in the near field of chiral plasmonic nanostructures. We calculate and visualize the degree of chirality to identify regions with relatively high values. This analysis leads to design principles for a simple utilization of chiral fields. We investigate planar geometries, which offer a convenient way to access the designated fields, as well as threedimensional nanostructures, which show a very high local optical chirality.
\end{abstract}

DOI: 10.1103/PhysRevX.2.031010

\section{INTRODUCTION}

An object that cannot be superimposed onto its mirror image is called chiral. When chiral objects (such as most biological molecules) interact with circularly polarized light, which is chiral itself, effects like circular dichroism or optical rotatory dispersion can occur [1]. In recent years, chiral plasmonic nanostructures have gained substantial interest [2-4]. Chiroptical effects that are several orders of magnitude higher than those for common biomolecules have been reported even in planar structures [5,6]. Novel fabrication techniques also enabled the analysis of bilayered [7-9] and three-dimensional [10-14] chiral nanostructures. Such structures exhibit not only a high amount of circular dichroism and optical rotatory dispersion, but they can also massively enhance the signals obtained from biological molecules [15-18], which in principle provides a method to detect, with a very high sensitivity, the handedness of a given molecule.

To quantify this enhancement, the so-called optical chirality $C$, which is a time-even pseudoscalar, can be used [19-21]:

$$
C(\vec{r})=\frac{1}{2}\left[\varepsilon_{0} \vec{E}(\vec{r}) \cdot \vec{\nabla} \times \vec{E}(\vec{r})+\frac{1}{\mu_{0}} \vec{B}(\vec{r}) \cdot \vec{\nabla} \times \vec{B}(\vec{r})\right],
$$

where $\vec{E}$ and $\vec{B}$ are the electric and magnetic fields, respectively. In the first discussion of this value in 1964, only mathematical aspects were analyzed. No physical meaning was assigned to that quantity [19]. Nevertheless, it has recently been shown that the optical chirality is correlated to the rate of excitation $A$ of a chiral molecule [22]:

$$
A \propto \alpha \omega U_{e}-\beta C,
$$

\footnotetext{
*giessen@physik.uni-stuttgart.de
}

Published by the American Physical Society under the terms of the Creative Commons Attribution 3.0 License. Further distribution of this work must maintain attribution to the author(s) and the published article's title, journal citation, and DOI.
Subject Areas: Metamaterials, Optics, Plasmonics

where $\omega$ and $U_{e}$ are the angular frequency and the local electric energy density of the surrounding field, respectively. $\alpha$ and $\beta$ denote material parameters describing the molecule [23]. The parameter $\beta$ is of special importance as it is of opposite sign for the two enantiomers of a handed molecule [24]. The difference in $A$ therefore depends on the optical chirality $C$ of the incident light. If one can increase this property at the location of the chiral molecule, the sensitivity for the detection of the handedness of the molecule can be enhanced.

We show that plasmonic nanostructures are suitable for this enhancement. While this has already been demonstrated in principle [15], we perform the first systematic comparison between different plasmonic nanostructures that leads the way to tailor such structures for specific applications such as enantiomeric sensing. Appropriate designs should provide not only a high enhancement of the optical chirality, but also large continuous regions where the fields with enhanced chirality are located and can be accessed, for example, by chiral liquids or molecules. We here work out several design principles that help to fulfill these requirements.

\section{RESULTS AND DISCUSSION}

To calculate the optical chirality, a simpler version of Eq. (1) can be deduced [22]:

$$
C(\vec{r})=-\frac{\varepsilon_{0} \omega}{2} \operatorname{Im}\left[\overrightarrow{\mathcal{E}}^{*}(\vec{r}) \cdot \overrightarrow{\mathcal{B}}(\vec{r})\right] .
$$

Note that $\overrightarrow{\mathcal{E}}$ and $\overrightarrow{\mathcal{B}}$ here denote the complex field amplitudes. We calculate the local enhancement of the optical chirality as

$$
\hat{C}(\vec{r})^{ \pm}:=\frac{C^{ \pm}}{\left|C_{\mathrm{CPL}}^{ \pm}\right|}
$$

for left-handed (LCP, +) and right-handed (RCP, -) circularly polarized light as incident waves. $C_{\mathrm{CPL}}^{ \pm}$are the values obtained for circularly polarized light without the nanostructure [25]. The sign of $C$ is preserved during this normalization, which helps to distinguish regions with 
different handedness of the local field. Afterward, we visualize the chiral fields via three-dimensional volume plots. Hereby, different nanostructures can be compared by the shape and the distribution of the regions with enhanced optical chirality. We use the same range for the color schemes in all plots, which allows for a direct comparison of different structures. Additionally, a rough comparison is possible by analyzing the minimum and maximum values gained for each structure. We use a median filter to smoothen the simulated data from numerical noise, which occurs in the form of single points with unnaturally high chirality values [26].

\section{A. Plasmonic structures for enantiomeric sensing}

First, we will discuss the general applicability of plasmonic nanostructures for enantiomeric sensing. By now, the most common method to detect the handedness of chiral molecules is a circular dichroism measurement. With this method, one illuminates the sample with leftand right-handed circularly polarized light and detects the difference signal of transmission or absorption [27]. In a more general scheme, one could use just two different light fields. For such measurements, the enantioselectivity is quantified by the dissymmetry factor $g$, which is defined as

$$
g:=\frac{2\left(A^{+}-A^{-}\right)}{A^{+}+A^{-}} .
$$

If the two light fields differ only in the sign of their local optical chirality, the dissymmetry factor is proportional to $-C / U_{e}$ [22]. This is, of course, the case for circularly polarized light. However, it has been demonstrated recently that the enantioselectivity can be enhanced using a partially reflecting mirror. The electric-field density is lowered at some spatial positions due to interference, while the optical chirality remains independent of position. This effect has been referred to as superchiral light as the dissymmetry factor exceeds the one obtained for circularly polarized light [20]. However, the relation between the total energy density of the field and the optical chirality remains independent of position, because, for regions with low electric energy density, the magnetic-energy density is increased [28]. Nevertheless, the excitation due to an external magnetic field is negligibly small and therefore irrelevant for enantiomeric sensing [22].

However, when we look at plasmonic nanostructures instead of the above-mentioned interference of plane waves, our analysis reveals that-depending on the polarization state of the incident light-different chiral fields can be induced. In general, the local optical chirality differs not only in sign but also in magnitude near the nanostructure. Additionally, the electric energy density can change when the incident polarizations excite different plasmonic modes. Of course, one could calculate the dissymmetry factor using Eq. (5). But using this formula would restrict the calculation to one specific chiral molecule, as the material constants $\alpha$ and $\beta$ have to be taken into account to obtain $A^{ \pm}$.

To obtain some general insight despite that issue, we analyze a superstructure composed of both of the enantiomers of one given chiral plasmonic structure (cf. Fig. 1). When one changes the polarization of the incident light, the optical chirality exhibits no local sign flips. Rather, for each spatial location near one enantiomer, there exists a corresponding location near the other where the fields feature the same electric energy density and opposite optical chirality. Then, the dissymmetry factor for two molecules located at these positions (which we refer to as $g^{*}$ ) becomes [29]

$$
g^{*} \propto-\frac{C^{+}-C^{-}}{U_{e}^{+}+U_{e}^{-}} .
$$

Note that only one of the enantiomers of the structure is actually involved in this calculation. We use the lefthanded enantiomer as it normally shows the stronger response to incident left-handed circularly polarized light.

In the following discussion, we carry out this kind of analysis for a helical structure, being one of the most intuitive examples for a chiral plasmonic nanostructure. Indeed, it has been demonstrated that such a structure exhibits very strong circular dichroism [30]. We used a gold helix with two windings, a diameter as well as a total height of $400 \mathrm{~nm}$, and a wire thickness of $80 \mathrm{~nm}$. This structure is resonant to incident light at a wavelength of $2.03 \mu \mathrm{m}$ when the handedness of the incident polarization matches the one of the helix. Therefore, one can expect a strong difference in response for the two circular polarization states.

Figure 2 depicts the calculated optical chirality for such helices. We show only the cases with matching incident polarization because, for the nonmatching configurations, the absolute values of optical chirality are too small to be visible in the three-dimensional map. The two enantiomers

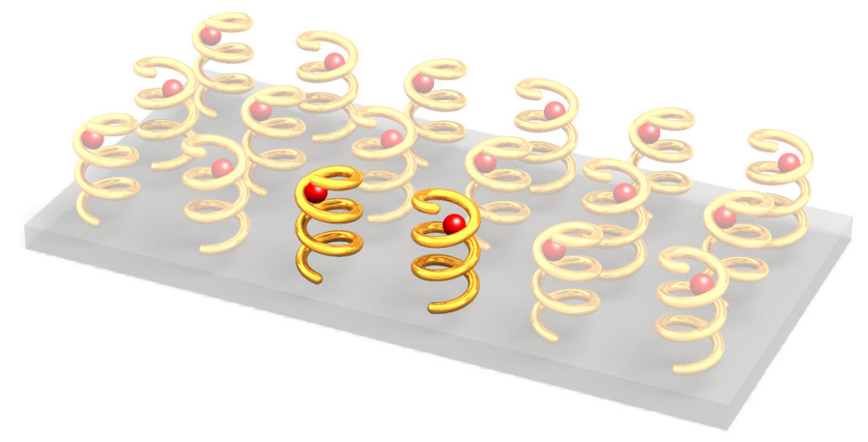

FIG. 1. To calculate the enantioselectivity in presence of chiral plasmonic structures, a modified dissymmetry factor $g^{*}$ is used. The definition requires a superstructure composed of both of the enantiomers of the single chiral nanostructure. Chiral probe molecules of one handedness (sketched as red spheres) are placed at corresponding positions at each of the enantiomers where the near fields differ only in the sign of optical chirality when the incident polarization is switched. 


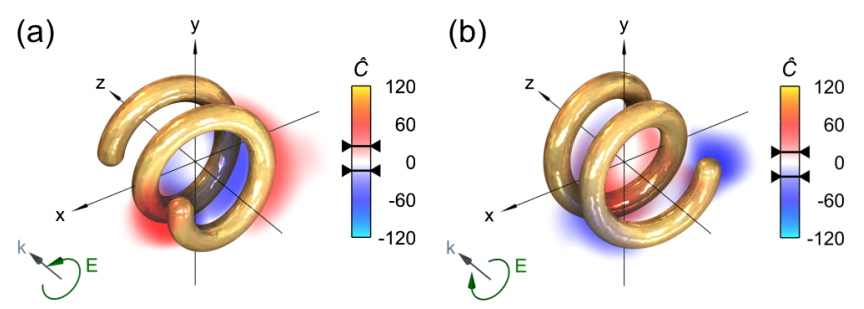

FIG. 2. Optical chirality enhancement for (a) a left-handed helix with left-handed circularly polarized light and (b) a right-handed helix with right-handed circularly polarized light at a wavelength of $2.03 \mu \mathrm{m}$. Diameter and height of the helix are $400 \mathrm{~nm}$ with a gold thickness of $80 \mathrm{~nm}$. Both combinations show enhanced optical chirality where the values of maximum and minimum enhancement are denoted by the black horizontal lines across the color bars. The regions with enhanced optical chirality are located at corresponding positions of the respective helix, but their signs flip. The pictures addressing the polarization state are taken from the detector's view; hence, the direction of the arrow indicates the handedness of the field vectors in space at a fixed time.

of the helix show symmetric distributions of enhanced optical chirality with respect to each other. More precisely, the values of optical chirality feature the same magnitude but opposite sign at corresponding positions. Therefore, the dissymmetry factor $g^{*}$ [cf. Eq. (6)] can be calculated.

The result is shown in Fig. 3(a) where we plot the enhancement $\hat{g}^{*}$ of $g^{*}$ with respect to the value $g$ obtained for circularly polarized light. This is carried out in the same way as the calculation of $\hat{C}$ [cf. Eq. (4)]. Note that this factor is not for the single helix, but rather for a combination of both the left-handed and the right-handed helices as depicted in Fig. 1. For a real application, one has to cover symmetric regions of both of these structures with the chiral sample.

We find a quite complicated distribution of $\hat{g}^{*}$ that is different from the one for $\hat{C}$ plotted in Fig. 2. This is due to (a)

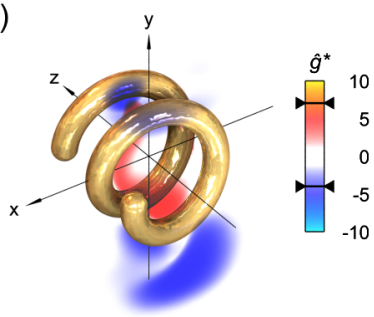

(b)

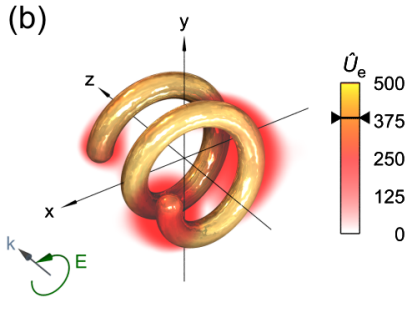

FIG. 3. (a) Enhancement of dissymmetry factor $\hat{g}^{*}$ near a plasmonic helix structure with respect to circularly polarized light. Superchiral light fields with up to 7 times higher enantioselectivity could be obtained. This value does not follow the enhancement of optical chirality directly, as (b) the enhancement of electric energy density $\hat{U}_{e}$ also enters the calculation. The electric energy density shows similar distributions as optical chirality, leading to more complicated shapes of the superchiral light fields. Note the different scale of the color bar related to the plot of $\hat{g}^{*}$ compared to the plots of $\hat{C}$ in Fig. 2. the contribution of the electric energy density to the dissymmetry factor which is also high at locations with strong optical chirality [cf. Fig. 3(b)]. As the electric-field enhancement is much stronger than optical chirality enhancement, the dissymmetry factor is in the end lower than for circularly polarized light at these positions. However, the calculation shows that there are regions where also the dissymmetry factor and therefore the enantioselectivity is increased. We have reached an enhancement factor of up to 7 for this model configuration. Note that the superstructure used for this analysis is achiral as it consists of both of the enantiomers of the chiral plasmonic helix. Therefore, we expect no chiroptical far-field response in the absence of chiral molecules, which allows for background-free measurements.

Of course, this is not a useful configuration for real sensing applications. Not only is the enhancement too small, but also the fabrication of the chosen structure is challenging due to the three-dimensional shape and the small dimensions. Also, the regions with enhanced enantioselectivity would be difficult to access. Yet, this example shows that chiral plasmonic nanostructures can indeed enhance the sensitivity of an enantiomer sensor.

In the following discussion, we will investigate different structures that overcome these problems. To keep to the general aim of this work, we decided to restrict our further analysis to the enhancement of optical chirality and leave the calculation of the dissymmetry factor to future work. Our results can be directly used for a simpler detection scheme in which the rate of excitation for just one incident polarization is measured directly. This scheme also allows for a distinction between the two enantiomers of that molecule due to the change of the sign of $\beta$ in Eq. (2). Our analysis of optical chirality enhancement will also suit other imaginable applications based on this quantity. As a consequence of the general approach, it is up to the readers to adopt our results to the needs of their specific applications.

\section{B. Planar nanostructures}

We start with an analysis of optical chirality enhancement by the gold gammadion structure introduced in Ref. [15] with respect to enantiomeric sensing. We use similar dimensions: $80 \mathrm{~nm}$ for both the width of the arms and the gaps, leading to a total width of $400 \mathrm{~nm}$, but a gold thickness of $20 \mathrm{~nm}$ instead of $100 \mathrm{~nm}$. For different sizes of the nanostructure, we expect the optical chirality to scale with the electric dipole moment of the particle plasmon, and hence with the volume of the individual nanoparticles. The structure is embedded in air. We calculate the fields at the fundamental plasmon resonance at $2.01 \mu \mathrm{m}$.

In contrast to the helix, the gammadion shows a similar behavior for both LCP and RCP as incident polarizations (cf. Fig. 4). We calculate enhancement factors for optical chirality in the range of 20 , which is comparable to the 

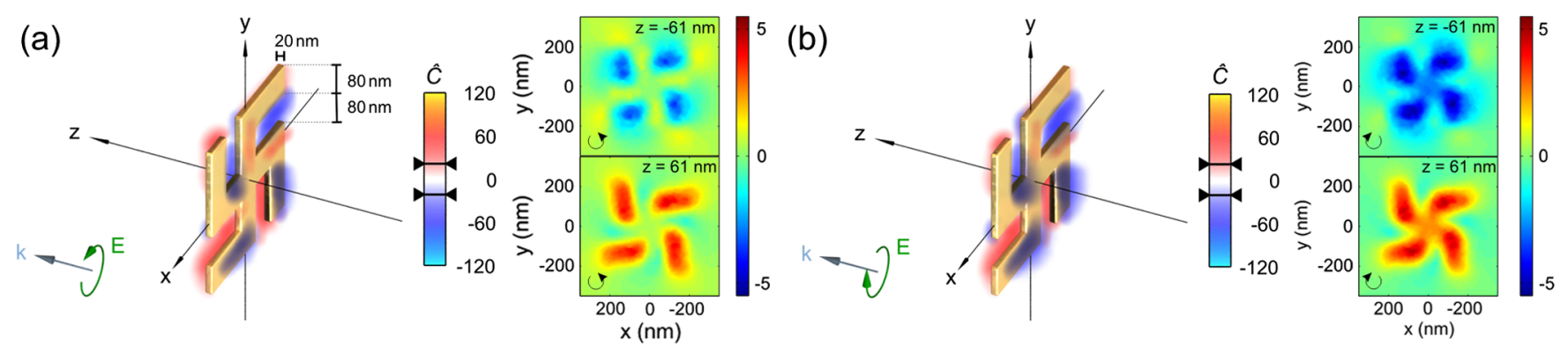

FIG. 4. Optical chirality enhancement by a planar gammadion structure illuminated with (a) LCP and (b) RCP at a wavelength of $2.01 \mu \mathrm{m}$. The structure exhibits similar shapes for the regions with enhanced chirality for both polarizations; only the values differ (as best seen in the two-dimensional slice views).

values obtained for the helix. As a major difference, the gammadion shows both positive and negative values of optical chirality with similar absolute values, while for the helix the values corresponding to light with matching polarization are higher [31].

The regions with locally enhanced optical chirality are mainly located at the front and the back of the four arms. In contrast, the regions with highest field enhancement can be found at the end of each arm and in the gaps beneath these ends (cf. Fig. 5). This result shows that such a nanostructure focuses the optical chirality at distinct points in space, similar to the field-enhancement effect. Nevertheless, the regions with highest enhancements differ for the two quantities. High field enhancement is therefore not sufficient to obtain a strong optical chirality as the polarization of the incident light is not maintained by the field-enhancement effect.

A drawback of the gammadion design is that regions with enhanced optical chirality are at the same position and have the same sign for both incident polarizations; only the absolute values differ. Therefore, a change of the incident polarization will result in only a small change of the optical chirality at a certain position, which renders this design
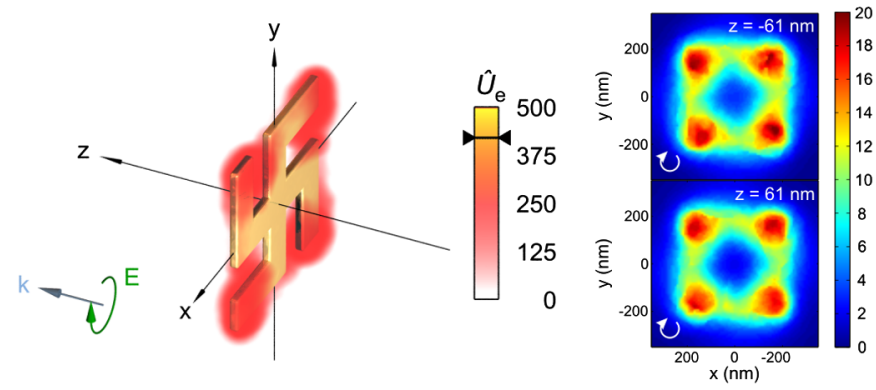

FIG. 5. Enhancement of the electric energy density $\hat{U}_{e}$ by the planar gammadion structure illuminated with right-handed circularly polarized light at a wavelength of $2.01 \mu \mathrm{m}$. As for the optical chirality, the electric energy density has been normalized by the value obtained for RCP without the presence of the nanostructure. Strongest enhancement can be found at the end of each arm and the nearby gaps. For LCP, the enhancement is similar in shape. impractical for any application using more than one light distribution. As additional disadvantages, the enhancement is discontinuous and overall quite small.

In Fig. 6 we show a more advanced planar nanostructure, namely, the nanospiral. Compared to the gammadion, the spiral features fewer sharp corners, which allows for smoother distributions of the regions with enhanced optical chirality. The two-armed design is chosen to operate at $1.84 \mu \mathrm{m}$ [32] with comparable dimensions ( $80 \mathrm{~nm}$ as the arm width with a gold thickness of $20 \mathrm{~nm}$ ). Each spiral features one and a half rotations, while the gammadion offers only one $90^{\circ}$ kink at each arm.

As a result, the two incident polarizations show different behavior, which can be explained by Fig. 7 where the induced current distributions for incident LCP and RCP light are plotted. The different polarizations excite different modes. Left-handed circularly polarized light excites currents in the outer part of the structure, which leads to the ringlike region with enhanced chirality, while the enhanced region for right-handed circularly polarized light is concentrated more in the center. Also, the signs of the chirality at the front and the back of the spiral along the $z$ direction change for the different polarizations.

This sign flip leads to a significant advantage over the gammadion: When one changes the polarization state of the incident light, the optical chirality changes in a much stronger fashion. This strong change aids any application that makes use of two different incident polarizations and analyzes a quantity influenced by the difference of optical chirality.

The plot of the difference in optical chirality enhancement $\Delta \hat{C}=\hat{C}_{+}-\hat{C}_{-}$shows quite uniform shapes [cf. Fig. 8(a)]. The regions with different polarity are clearly divided by the structure itself. For many applications such as enantiomer sensing or vibrational and rotational optical activity measurements, one wants to use only one particular polarity, as the second polarity would possibly lessen the signal. This use of a particular polarity can be simply accomplished for the nanospiral, as one has only to ensure that the access is limited to one side of the structure. When the structure is fabricated on top of a substrate, this requirement is automatically fulfilled [cf. Fig. 8(b)]. 

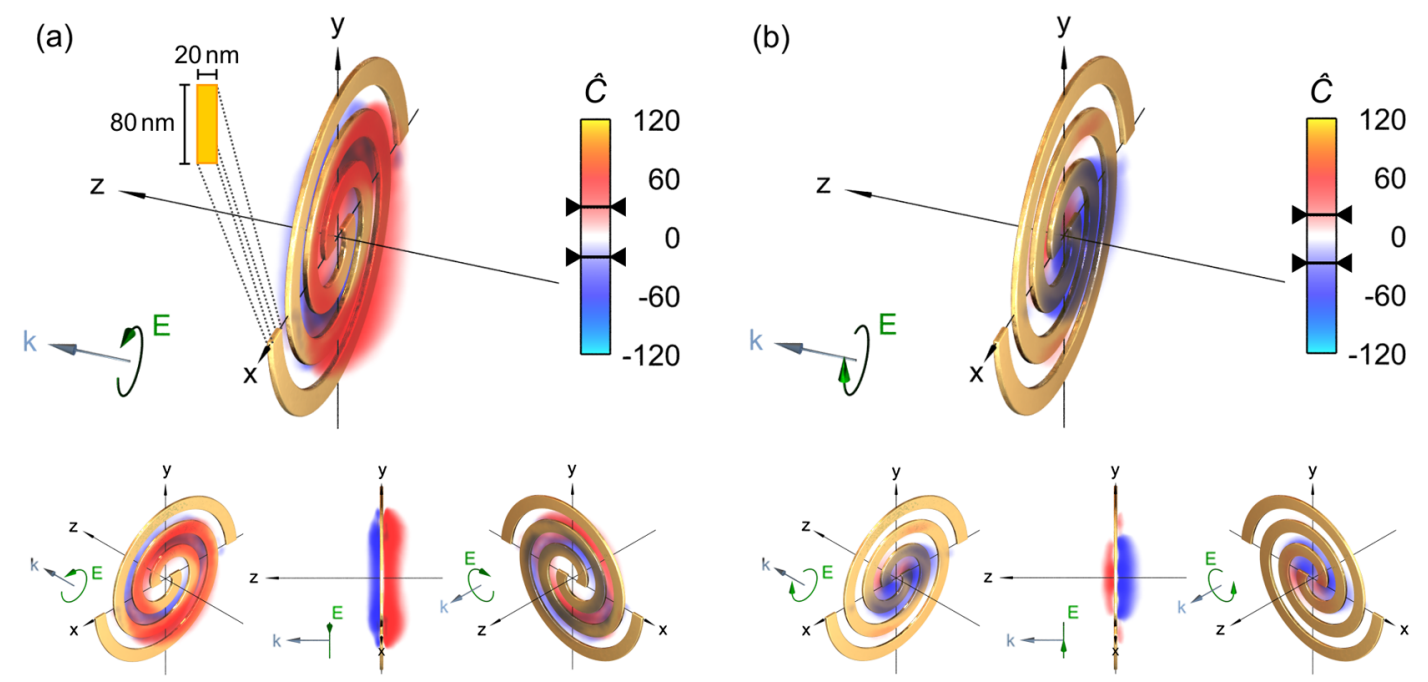

FIG. 6. Optical chirality enhancement by a two-armed gold nanospiral with (a) LCP and (b) RCP as incident polarizations at a wavelength of $1.84 \mu \mathrm{m}$. The two polarizations differ significantly in their near-field response. The smaller pictures on the bottom of (a) and (b) show the scenario from different angles. The enhancement of the optical chirality for the nanospiral is up to 50\% higher than for the gammadion, which can be understood by the fact that the spiral is bent everywhere.

It is important to note that all structures analyzed thus far are achiral, as planar structures cannot possess structural chirality in a three-dimensional space. Nevertheless, the comparison between gammadion and nanospiral shows that stronger optical chirality as well as a more uniform enhancement can be obtained by a structure with a stronger twist. This twist can be referred to as planar chirality. Although the chiral far-field response of such structures occurs only in the presence of a substrate [5], the chiral near-field enhancement is possible even without the substrate, as shown in our simulations.

A drawback of structures with only planar chirality arises when the response for the two different incident polarizations is compared. Although the absolute values of $\hat{C}$ are up to $50 \%$ higher than those of the gammadion, at the spatial points where the chirality enhancement for LCP is strong, the contribution of RCP is negligibly small, and vice versa. This correlation limits the benefit of switching between the two polarizations. To overcome this limitation,

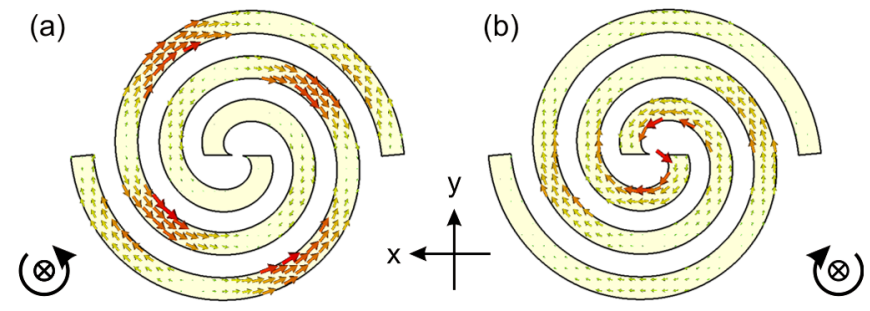

FIG. 7. Current distributions inside the nanospiral for incident (a) LCP and (b) RCP light at wavelength $1.84 \mu \mathrm{m}$. The two polarizations excite different modes. The current distributions correspond to the regions with enhanced optical chirality as identified in Fig. 6. one can use three-dimensional structures, which can exhibit intrinsic structural chirality.

\section{Three-dimensional chiral nanostructures}

The helix discussed in Sec. II A provides the desired features in principle. Although the response for any combination of helix and incident light with opposite handedness is very small, the strongest enhancement can be found at spatial locations similar to those for the matching polarization but with different sign. But, as already discussed, such a structure would be impractical for real applications because of the small and discontinuous response as well as fabrication difficulties.

To overcome these problems, we propose a much simpler bilayered structure which mimics a helix. We call this structure a chiral oligomer [33-37]. The design is sketched in Fig. 9: Each layer consists of three gold disks which are (a)

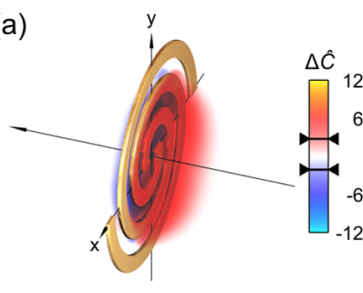

(b)

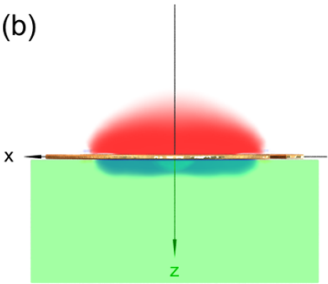

FIG. 8. Difference $\Delta \hat{C}=\hat{C}_{+}-\hat{C}_{-}$of the chirality enhancement of the nanospiral. (a) The side view shows that the distribution of the enhanced regions is continuous, while the structure separates the parts with different signs. (b) In the presence of a substrate, the access is automatically limited to one polarity. Note that this picture is only a sketch, as the calculation was performed without the substrate. 


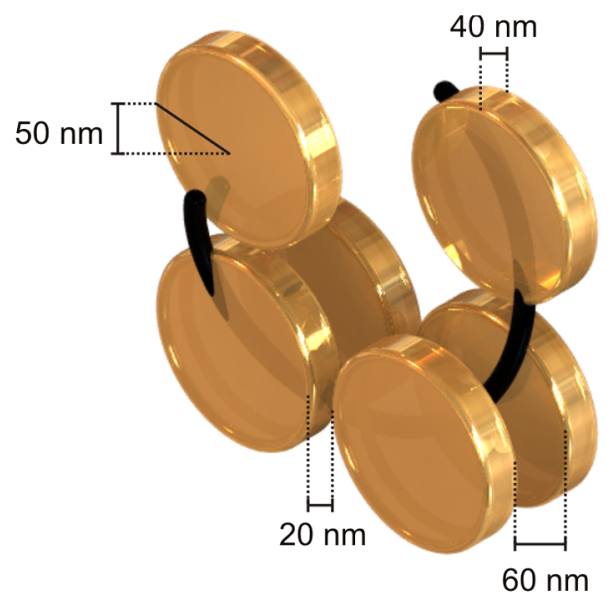

FIG. 9. Sketch of the chiral plasmonic oligomer. The arrangement of the gold disks mimics the chiral structure of a lefthanded helix.

arranged in an L-shaped configuration. The second layer is twisted by $90^{\circ}$ to emulate the chiral layout of a helix. The stacking of several layers with planar structures is a well-established technique in nanofabrication [38]. Additionally, the usage of small disks as building blocks allows for tuning of the operation wavelength to lower values: The resonant operating wavelength is governed by the particle plasmon resonance of the individual gold nanodisks, modified by plasmon hybridization due to strong near-field coupling $[39,40]$. This effect is demonstrated by the dimensions chosen in Fig. 9, which lead to a structure with maximum circular dichroism in the near infrared at $900 \mathrm{~nm}$.

Figure 10 shows the optical chirality enhancement induced by the chiral oligomer. Like in the case of the helix, we obtain a much stronger result when the handedness of both the structure and the incident light coincide. Compared to the gammadion, the response of $\hat{C}$ is about 5 times higher for LCP [Fig. 10(a)], while, for incident RCP [Fig. 10(b)], still comparable values occur. The highest absolute values for both polarizations are located at the same spatial positions, which leads to regions with $\Delta \hat{C}$ of more than 100 [Fig. 10(c)]. The analysis of the distribution of the regions with enhanced chirality reveals that (besides some small areas near the surfaces of the disks) a continuous region with consistent polarity is located around the two disks at the bottom left of the oligomer. Figures 10(d)-10(f) show slices through the point with highest chirality enhancement (located in the gap between the top and the bottom left disk of the front layer). We consider that this strong effect is due to near-field enhancement, which is highest in the small
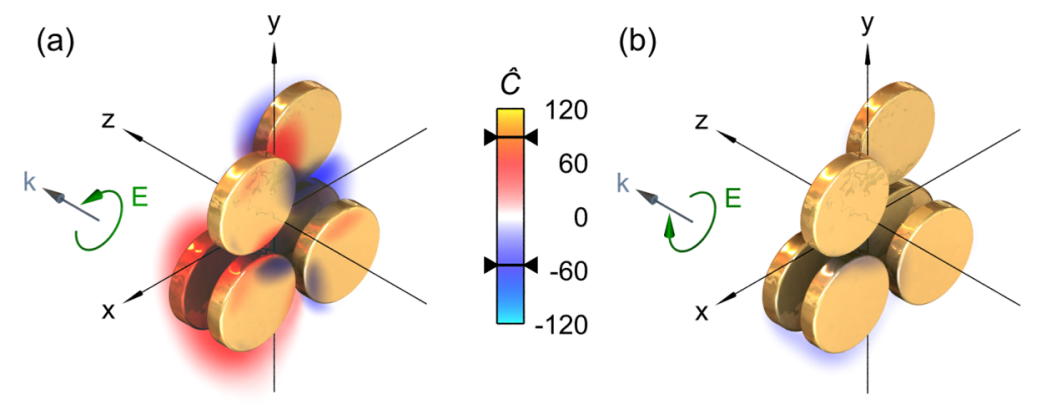

(d)

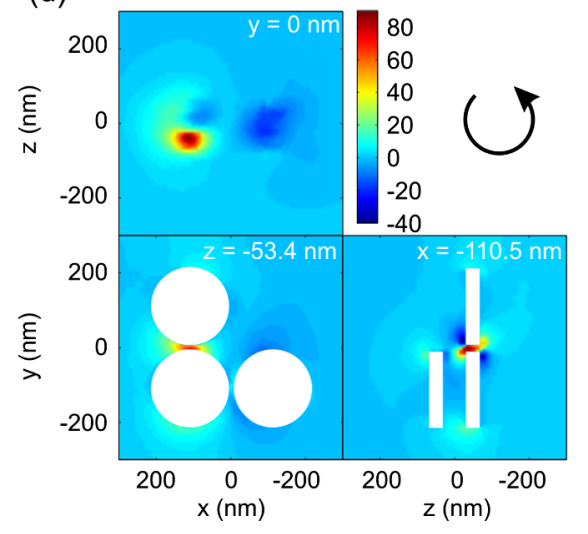

(e)

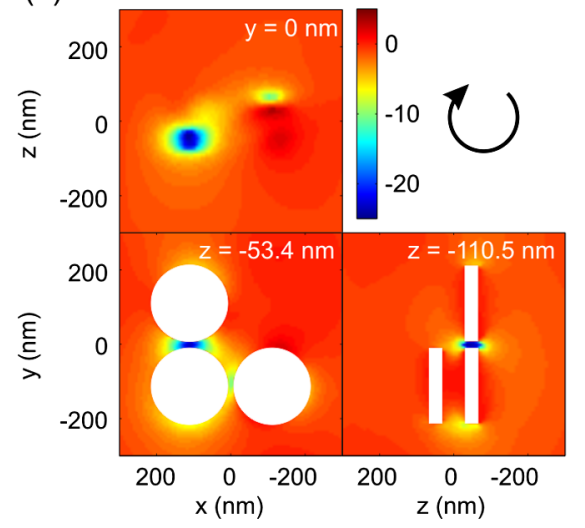

(c)

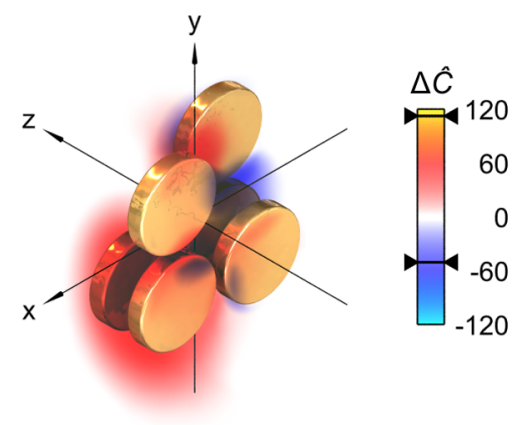

(f)

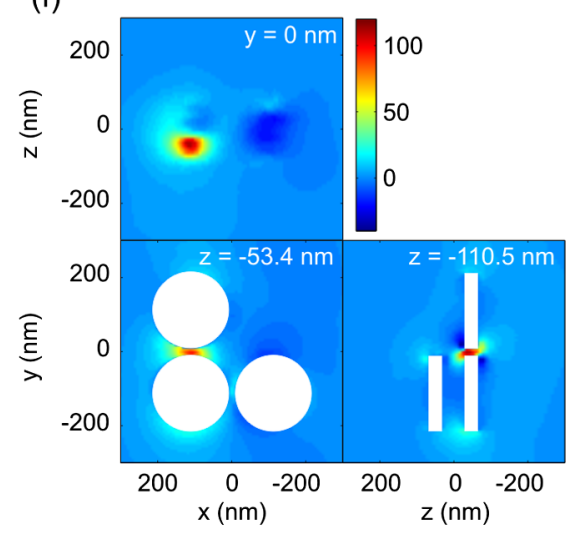

FIG. 10. Optical chirality enhancement of the chiral oligomer for incident (a),(d) LCP and (b),(e) RCP light at a wavelength of $900 \mathrm{~nm}$. The spatial points with strongest enhancement can be found on similar spatial locations but exhibit different signs, which leads to (c),(f) a very strong difference $\Delta \hat{C}$. (d)-(f) Slices through the point between the top and bottom left disk of the front layer show that the region with strongest enhancement is located in the gap between these two disks. 

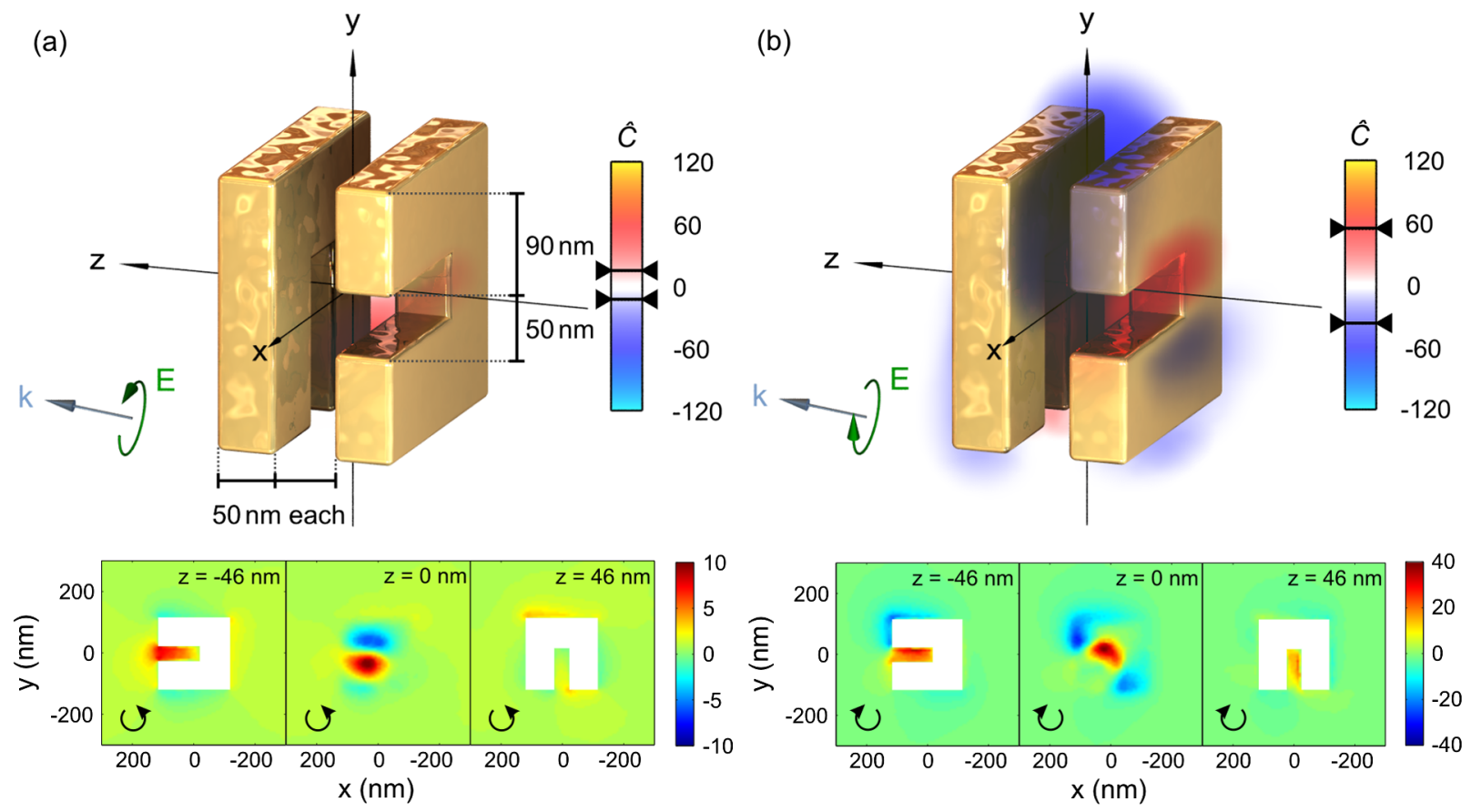

FIG. 11. Optical chirality enhancement induced by the stereometamaterial with (a) LCP and (b) RCP as incident polarizations at wavelength $1.34 \mu \mathrm{m}$. Note that the enhancement is strongest when the handedness of the light is opposite to the handedness of the structure. The strongest enhancement can be observed in the middle of the layer between the two split-ring resonators.

gaps between the disks. Unfortunately, access to these strongly enhanced chiral fields is more challenging than in the planar case, since the structure has to be embedded in a dielectric matrix.

The third dimension offers many more possibilities for chiral structures than the two-dimensional case. In Fig. 11 we obtain the optical chirality for stacked and twisted plasmonic split-ring resonators. The chiral far-field response of these so-called stereometamaterials [41] is well known $[42,43]$. The dimensions were chosen to fit the geometry introduced in Ref. [41], which leads to a wavelength with highest circular dichroism of $1.34 \mu \mathrm{m}$. Interestingly, we find that, for this left-handed structure, the strongest chirality enhancement is obtained not for LCP [Fig. 11(a)], but for RCP incident light [Fig. 11(b)]. For both polarizations, positive chirality values are obtained in the gap while negative values occur in the surrounding medium. The strength of the enhancement is between the values of the planar structures and the chiral oligomer. The strongest enhancement is not located in the gap of one split-ring resonator but in the layer between the two resonators. Additional degrees of freedom such as the twist angle could be used to tune this behavior.

\section{CONCLUSIONS}

We have explored the chiroptical near-field response of different planar as well as three-dimensional plasmonic nanostructures and compared the resulting enhancement of optical chirality. Using the example of the helix, we have shown in principle that plasmonic nanostructures can be utilized to enhance the enantioselectivity locally.

When comparing different structures, there is a huge parameter space to explore, and the optical chirality enhancement is nontrivial to predict. Therefore, we give as a conclusion some design principles that we extracted from our present analysis to match the demands of different applications. If simple access to a continuous region of enhanced optical chirality is needed, a planar structure with a strong twist and ideally without sharp corners should be used. A versatile example for such a design is our plasmonic nanospiral. To obtain large differences of the optical chirality, we recommend three-dimensional chiral structures that should be as compact as possible. The chiral oligomer, which is of that type, showed the highest chirality enhancement we could obtain theoretically. For easier fabrication, multilayered structures should be preferred.

We have also demonstrated that local optical chirality is a new way to look at chiral structures. A strong chiral response in the far field - the strongest circular dichroism from our selected structures could be obtained for the helix-will not automatically generate fields with the strongest local optical chirality. Moreover, even achiral structures can lead to fields with high optical chirality. We found that near-field enhancement and twisting should be combined, although the highest optical chirality will not automatically occur at regions with highest electric energy density. To understand this, additional research is needed to 
work out the origin of the enhancement of the optical chirality for the different structures. This future work will also help to improve our design principles and thus to further optimize the suggested designs or to create new designs for enhanced chiral interaction [44] and enantiomeric sensing techniques.

\section{ACKNOWLEDGMENTS}

The authors thank Adam Cohen and Thomas Weiss for helpful discussions and Sven Hein for help with the visualization. This work was financially supported by BMBF (Nos. 13N9048 and 13N10146), DFG (Nos. SPP1391, FOR557, and GI 269/11-1), and Baden-Württemberg Stiftung. D. D. additionally acknowledges support by the Carl-Zeiss-Stiftung. This work was supported by the German Research Foundation (DFG) within the funding program Open Access Publishing.

[1] L.D. Barron, Molecular Light Scattering and Optical Activity (Cambridge University Press, Cambridge, England, 2004).

[2] A. J. Mastroianni, S. A. Claridge, and A.P. Alivisatos, Pyramidal and Chiral Groupings of Gold Nanocrystals Assembled Using DNA Scaffolds, J. Am. Chem. Soc. 131, 8455 (2009).

[3] Z. Fan and A. O. Govorov, Plasmonic Circular Dichroism of Chiral Metal Nanoparticle Assemblies, Nano Lett. 10, 2580 (2010).

[4] A. Guerrero-Martínez, J. L. Alonso-Gómez, B. Auguié, M. M. Cid, and L. M. Liz-Marzán, From Individual to Collective Chirality in Metal Nanoparticles, Nano Today 6, 381 (2011).

[5] M. Kuwata-Gonokami, N. Saito, Y. Ino, M. Kauranen, K. Jefimovs, T. Vallius, J. Turunen, and Y. Svirko, Giant Optical Activity in Quasi-Two-Dimensional Planar Nanostructures, Phys. Rev. Lett. 95, 227401 (2005).

[6] V. K. Valev, N. Smisdom, A. V. Silhanek, B. De Clercq, W. Gillijns, M. Ameloot, V. V. Moshchalkov, and T. Verbiest, Plasmonic Ratchet Wheels: Switching Circular Dichroism by Arranging Chiral Nanostructures, Nano Lett. 9, 3945 (2009).

[7] A. V. Rogacheva, V. A. Fedotov, A. S. Schwanecke, and N. I. Zheludev, Giant Gyrotropy Due to ElectromagneticField Coupling in a Bilayered Chiral Structure, Phys. Rev. Lett. 97, 177401 (2006).

[8] M. Decker, M. Ruther, C. E. Kriegler, J. Zhou, C. M. Soukoulis, S. Linden, and M. Wegener, Strong Optical Activity from Twisted-Cross Photonic Metamaterials, Opt. Lett. 34, 2501 (2009).

[9] R. Zhao, L. Zhang, J. Zhou, T. Koschny, and C. Soukoulis, Conjugated Gammadion Chiral Metamaterial with Uniaxial Optical Activity and Negative Refractive Index, Phys. Rev. B 83, 035105 (2011).

[10] J. K. Gansel, M. Thiel, M. S. Rill, M. Decker, K. Bade, V. Saile, G. von Freymann, S. Linden, and M. Wegener, Gold Helix Photonic Metamaterial as Broadband Circular Polarizer, Science 325, 1513 (2009).
[11] A. Radke, T. Gissibl, T. Klotzbücher, P. von Braun, and H. Giessen, Three-Dimensional Bichiral Plasmonic Crystals Fabricated by Direct Laser Writing and Electroless Silver Plating, Adv. Mater. 23, 3018 (2011).

[12] A. Guerrero-Martínez, B. Auguíe, J. L. Alonso-Gómez, Z. Džolić, S. Gómez-Graña, M. Žinić, M. M. Cid, and L. M. Liz-Marzán, Intense Optical Activity from ThreeDimensional Chiral Ordering of Plasmonic Nanoantennas, Angew. Chem. 123, 5613 (2011).

[13] E. Helgert, E. Pshenay-Severin, M. Falkner, C. Mensel, C. Rockstuhl, E.-B. Kley, A. Tünnermann, F. Lederer, and T. Pertsch, Chiral Metamaterial Composed of ThreeDimensional Plasmonic Nanostructures, Nano Lett. 11, 4400 (2011).

[14] A. Kuzyk, R. Schreiber, Z. Fan, G. Pardatscher, E.-M. Roller, A. Högele, C. Simmel, A. O. Govorov, and T. Liedl, DNA-Based Self-Assembly of Chiral Plasmonic Nanostructures with Tailored Optical Response, Nature (London) 483, 311 (2012).

[15] E. Hendry, T. Carpy, J. Johnston, M. Popland, R. V. Mikhaylovskiy, A.J. Lapthorn, S. M. Kelly, L.D. Barron, N. Gadegaard, and M. Kadodwala, Ultrasensitive Detection and Characterization of Biomolecules Using Superchiral Fields, Nature Nanotech. 5, 783 (2010).

[16] A. O. Govorov, Z. Fan, P. Hernandez, J. M. Slocik, and R. R. Naik, Theory of Circular Dichroism of Nanomaterials Comprising Chiral Molecules and Nanocrystals: Plasmon Enhancement, Dipole Interactions, and Dielectric Effects, Nano Lett. 10, 1374 (2010).

[17] A. O. Govorov, Plasmon-Induced Circular Dichroism of a Chiral Molecule in the Vicinity of Metal Nanocrystals. Application to Various Geometries, J. Phys. Chem. C 115, 7914 (2011).

[18] V. A. Gérard, Y.K. Gun'ko, E. Defrancq, and A.O. Govorov, Plasmon-Induced CD Response of Oligonucleotide-Conjugated Metal Nanoparticles, Chem. Commun. (Cambridge) 47, 7383 (2011).

[19] D. M. Lipkin, Existence of a New Conservation Law in Electromagnetic Theory, J. Math. Phys. (N.Y.) 5, 696 (1964).

[20] Y. Tang and A. E. Cohen, Enhanced Enantioselectivity in Excitation of Chiral Molecules by Superchiral Light, Science 332, 333 (2011).

[21] A. G. Smart, A Mirror Gives Light an Extra Twist, Phys. Today 64, No. 6, 16 (2011).

[22] Y. Tang and A.E. Cohen, Optical Chirality and Its Interaction with Matter, Phys. Rev. Lett. 104, 163901 (2010).

[23] In Eq. (2), $\alpha$ is the imaginary part of the electric polarizability, while $\beta$ is the imaginary part of the mixed electric-magnetic polarizability.

[24] D. P. Craig and T. Thirunamachandran, New Approaches to Chiral Discrimination in Coupling between Molecules, Theor. Chem. Acc. 102, 112 (1999).

[25] For details about the calculations and the simulation environment, see Supplemental Material at http://link.aps.org/ supplemental/10.1103/PhysRevX.2.031010.

[26] See Supplemental Material at http://link.aps.org/ supplemental/10.1103/PhysRevX.2.031010 for detailed information about the filtering process. 
[27] Circular Dichroism: Principles and Applications, edited by N. Berova, K. Nakanishi, and R. W. Woody (WileyVCH, New York, 2000), 2nd ed.

[28] K. Y. Bliokh and F. Nori, Characterizing Optical Chirality, Phys. Rev. A 83, 021803(R) (2011).

[29] For the calculation of $g^{*}$ and a more detailed discussion of this model, see Supplemental Material at http://link .aps.org/supplemental/10.1103/PhysRevX.2.031010.

[30] J. K. Gansel, M. Wegener, S. Burger, and S. Linden, Gold Helix Photonic Metamaterials: A Numerical Parameter Study, Opt. Express 18, 1059 (2010).

[31] See Supplemental Material at http://link.aps.org/ supplemental/10.1103/PhysRevX.2.031010 for a tabular comparison of these values.

[32] See Supplemental Material at http://link.aps.org/ supplemental/10.1103/PhysRevX.2.031010 for an explanation of how we chose the wave lengths at which we calculated the optical chirality.

[33] J. A. Fan, C. Wu, K. Bao, J. Bao, R. Bardhan, N. J. Halas, V. N. Manoharan, P. Nordlander, G. Shvets, and F. Capasso, Self-Assembled Plasmonic Nanoparticle Clusters, Science 328, 1135 (2010).

[34] M. Hentschel, M. Saliba, R. Vogelgesang, H. Giessen, A. P. Alivisatos, and N. Liu, Transition from Isolated to Collective Modes in Plasmonic Oligomers, Nano Lett. 10, 2721 (2010).

[35] J. B. Lassiter, H. Sobhani, J. A. Fan, J. Kundu, F. Capasso, P. Nordlander, and N.J. Halas, Fano Resonances in
Plasmonic Nanoclusters: Geometrical and Chemical Tunability, Nano Lett. 10, 3184 (2010).

[36] M. Hentschel, D. Dregely, R. Vogelgesang, H. Giessen, and N. Liu, Plasmonic Oligomers: The Role of Individual Particles in Collective Behavior, ACS Nano 5, 2042 (2011).

[37] M. Hentschel, M. Schäferling, T. Weiss, N. Liu, and H. Giessen, Three-Dimensional Chiral Plasmonic Oligomers, Nano Lett. 12, 2542 (2012).

[38] N. Liu, H. Guo, L. Fu, S. Kaiser, H. Schweizer, and H. Giessen, Three-Dimensional Photonic Metamaterials at Optical Frequencies, Nature Mater. 7, 31 (2007).

[39] N. Liu and H. Giessen, Coupling Effects in Optical Metamaterials, Angew. Chem. 49, 9838 (2010).

[40] N. J. Halas, S. Lal, W. Chang, S. Link, and P. Nordlander, Plasmons in Strongly Coupled Metallic Nanostructures, Chem. Rev. 111, 3913 (2011).

[41] N. Liu, H. Liu, S. Zhu, and H. Giessen, Stereometamaterials, Nature Photon. 3, 157 (2009).

[42] H. Liu, J. X. Cao, S. N. Zhu, N. Liu, R. Ameling, and H. Giessen, Lagrange Model for the Chiral Optical Properties of Stereometamaterials, Phys. Rev. B 81, 241403 (2010).

[43] D. A. Powell, K. Hannam, I. V. Shadrivov, and Y.S. Kivshar, Near-Field Interaction of Twisted Split-Ring Resonators, Phys. Rev. B 83, 235420 (2011).

[44] D. E. Gómez, K. C. Vernon, and T. J. Davis, Symmetry Effects on the Optical Coupling Between Plasmonic Nanoparticles with Applications to Hierarchical Structures, Phys. Rev. B 81, 075414 (2010). 